How to Cite

Hossain, M. (2018). Cultural and individual differences in comprehension of sports metaphors. International Journal of

Humanities, Literature \& Arts, 1(1), 25-30. https://doi.org/10.31295/ijhla.v1n1.30

\title{
Cultural and Individual Differences in Comprehension of Sports Metaphors
}

\author{
Musharrat Hossain \\ Department of English and Modern Languages North South University, Bashundhara, Dhaka-1212, Bangladesh, \\ Email: musharrat.hossain@northsouth.edu
}

\begin{abstract}
This paper attempts to find out the effect of controlled input and rate of retention of sports metaphors by Bengali speakers of English over a period of 4 weeks. Metaphors are non-literal expressions that contain culturespecific information that does not correspond with what they state (Cooper, 1998). It has been a constant site of struggle for L2 speakers of English to understand metaphors that readily mean something for a native speaker of English. A metaphor brings up an image in the native speaker's mind but even an imagable idiom some time fails to conjure up images in the L2 speaker's mind. (Boers and Deemecheleer, 2001). To address this question, a four-week long research is conducted in order to find out the acquisition and retention of 10 sports metaphors from the specific domains of Baseball and American Football. To conduct the study, The Boers (2004) model have been followed. That is: i) by making the figurative language literal ii) by employing learners' problem-solving skills for retention of the metaphors, and iii) grouping idioms under a broad metaphoric theme for clearer understanding. The results show that controlled and repeated input along with factors like motivation and frequency have instigated into acquisition and retention of the sports metaphors by the participants. It also shows that metaphor acquisition is a socio-cognitive process where a learner learns in the context by analyzing contextual clues and by exchanging between several mental domains.

Keywords---competence, cross-cultural, language acquisition, language domains, metaphor.
\end{abstract}

\section{Introduction}

One of the reasons why metaphors are often difficult to teach and/or learn is that they are non-literal expressions that contain culture-specific information. Metaphors are often associated with a culture-specific event/ information and they often do not mean what they state (Cooper, 1998). To access the meaning of a metaphor, it is important to decode the cultural information that may not be readily available to cultural outsiders.

Even though metaphors are almost always readily understood by native speakers of English, they become the site of the frequent complaint and struggle for non-native speakers of English, especially those who come from a significantly different cultural background. Sometimes learners from two culturally similar societies may not readily access metaphors of the other language. For example, Boers \& Demecheleer (2001) showed in their study that among 78 french L2 speakers, only 56\% could guess the meaning of idioms associated with "hat", whereas almost $60 \%$ could not guess the meaning of idioms associated with "sleeve". It is important to remember that French speakers have more cultural similarities with English speakers.

Bengali L2 speakers have fewer similarities with the English speaking societies, therefore, metaphors of specific domains may present even greater difficulties for them. This paper attempts to measure to what extent Bengali L2 speakers who are graduate students of Engineering can understand sports metaphors frequently used in American discourse in and out of context.

It is worthwhile to mention that, metaphors are usually multi-word units (MWU). An MWU can be defined as a fixed and recurrent pattered sanctioned by usage (Grant \& Bauer, 2004). Idioms are also usually MWUs which relate back to a specific domain but are used in multiple domains based on their metaphorical potential and use. In the past, the most commonly accepted 5 point criteria for metaphor/ idiomaticity were what Fernando \& Flavell (1981) had provided us with. They are:

ISSN 2632-9441

Received Jan 10, 2018 / Accepted Jun 20, 2018 / Published Jul 05, 2018 
a) The meaning of an idiom is not the result of the compositional function of its constituents

b) An idiom is a unit that that either has a homonymous literal counterpart or individual constitution that are literal, though the expression as a whole will not be interpreted literally

c) Idioms are transformationally deficient in one way or another

d) Idioms constitute set expression in a given language

e) Idioms are institutionalized.

The criteria have been redefined by and narrowed down by linguistics in various ways. It has been later defined as an imagable idiom that tend to call up a conventional scene in the native speakers mind Boers \& Demecheler (2001) or a set of MWU whose meaning cannot be predicted from its constituent parts (Grant \& Bauer 2004) an also as a bridge that brings into contact two or more different domains of knowledge - a source domain and a target domain (Lakoff \& Johnson, 1980).

For the study, I have chosen two specific domains of American Football and Baseball, and a list of 10 idioms related to those specific sports. I will have $8 \mathrm{~L} 2$ speakers of Bengali (male) living in the United States guess the meaning of the idioms out of context and then in context and measure if there will be the difference between the two sets. I anticipate some exceptional outcome based on the motivation of the speakers due to their interest in sports. Each individual participant will show a different trajectory in understanding and acquire the metaphors due to factors like motivation, interest, familiarity, etc. To measure that, I will first give a questionnaire in attempting to find out their interest in specific sports.

According to Boers (2004), metaphors are not only a tool for organizing figurative language input, it also increases the strategies related to imaging and concreteness for second language speakers. Some of the ways of teaching metaphors to SL learners, according to him, are i) by making the figurative language literal (or original), ii) by employing learners' problem-solving skills for retention of the metaphors, and iii) grouping idioms under a broad metaphoric theme for clearer understanding. I will apply all three of these strategies to measure the acquisition of metaphors of my participants.

The hypothesis for the study is that the understanding of the idioms will increase once they are presented in context and their a connection to the real world is explained. Through this process, once the participants are aware of the conventions of the specific sports domain, they will be able to understand the metaphors related to that sports in another conversational context. It is predicted that the participants will have easier access to Baseball metaphors for the conventional similarities of it with Cricket, sports, a popular sport in the Indian subcontinent.

\section{Research Method}

For this study, I chose 8 adult male participants aged between 24-30. All of my participants were engineering graduate students from Bangladesh and had been studying at Oklahoma State University for less than a year. Since they were doing graduate research work at a US university, it was safe to assume that all of them have at least above average proficiency in English. I began the study with a questionnaire, where the participants were to fill out some general information, like age, years of studying English, duration of stay in the United States, and favorite sports. After that, I gave them a questionnaire containing 5 metaphors each from the specific domains of American Football and Baseball, a total of ten metaphors. It was measured how many of metaphors they could understand correctly when presented out of context. I called this a metaphor access test or MAT. The metaphors that were given to them were: i) major league, ii) go to bat for someone, iii) the home stretch, iv) off base, v) hard hitter, vi) dancing at the end zone, vii) a running back viii) to score a touchdown, ix) kick off and $\mathrm{x}$ ) to hold the line. After that, I sat down with them and showed them youtube clips from a baseball game and a football game.

After one week, I met with the group again and handed out newspaper/ literary excerpts, and lyrics of a song containing those expressions in a metaphorical way. I, then, again discussed the spots conventions with them and had them connect those expressions with their metaphorical use. Two weeks later, I gave them the same MAT again to measure any changes of their understanding of the same metaphors that were given to them earlier, most of which they were previously unfamiliar with.

The metaphorical uses of the expressions have been taken from Corpus of Contemporary American English (COCA) and the frequency had been recorded. The participants were given printed copies of the newspaper and literary excerpts, and lyrics from the song. The result is shown in a graph for each of the participants and for the whole group. The results for the individual participants is considered crucial to analyze individual interest and motivation and how those factors may or may not affect acquisition of domain-specific metaphors. 


\section{Results and Analysis}

The results of the study showed that all of the participants had an increased understanding of sports metaphors from MAT-1 to MAT-2. The individual results differed based on their familiarity with the sports.

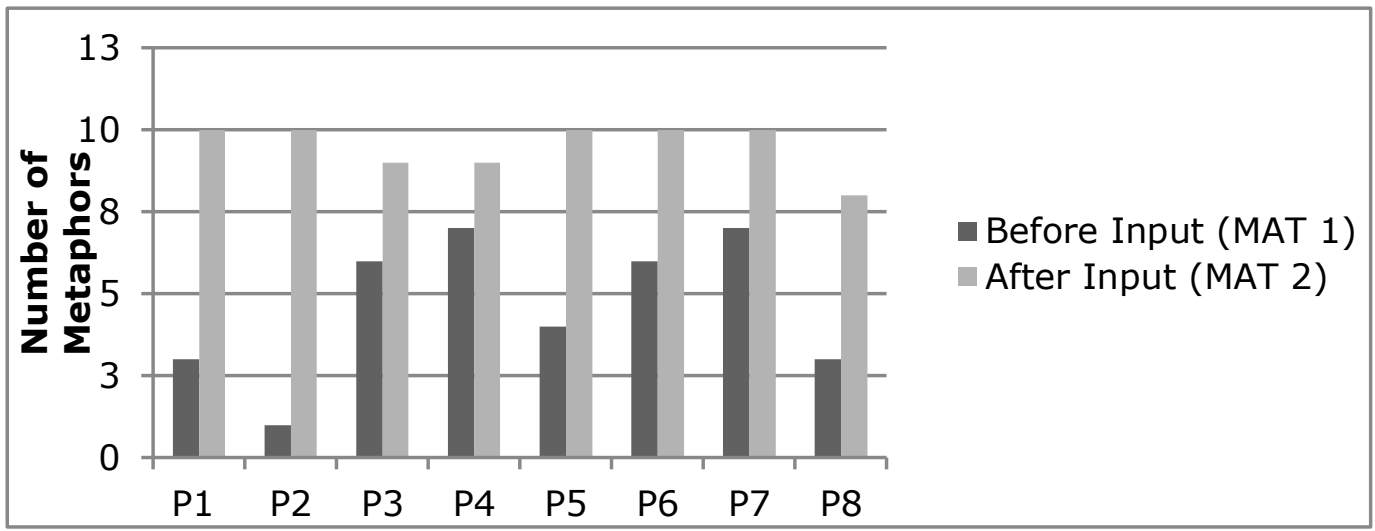

Figure 1. Individual acquisition of metaphors

Figure 1 shows the overall development in both the specific domains of Baseball and Football for the individual learners. From the data gathered, we see that there is an overall growth of $49 \%$ on average by the participants. However, in the usage-based theoretical framework, it is believed that averaging data leads to losing out on valuable usage data. This project, therefore, will zoom into the two individual domains and look into the data in detail. We will also zoom into some of the participants and attempt to find out meaningful information about their process of acquisition of sports metaphors.

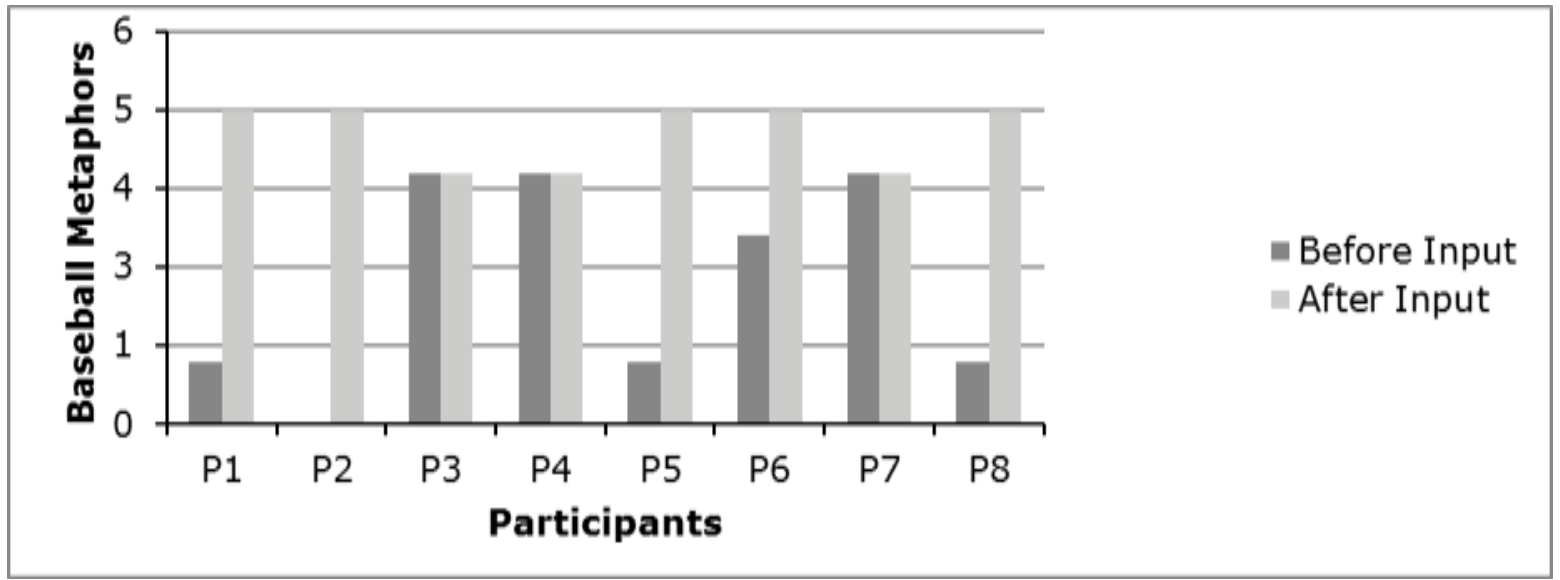

Figure 2. Individual acquisition of baseball metaphors

Figure 2 zooms into the Baseball domain and looks at development in that domain by the individual participant. In this section, we see that each participant is showing different levels of background knowledge and different levels of acquisition of the metaphors. 5 out of 8 participants successfully predict the meanings of all 5 of the metaphors after in input. Before the input, one of them showed no comprehension of the metaphors, 3 of them showed comprehension for 1 metaphor out of five.

The football domain shows a different picture. As assumed differently earlier, the participants already show higher understanding of football metaphors than baseball before any input was given to them. 4 out of 8 participants could correctly predict the meaning of the metaphors. After the input, 7 out of 8 participants showed an understanding of all the metaphors. The results of the football metaphors are described in Figure 3. 


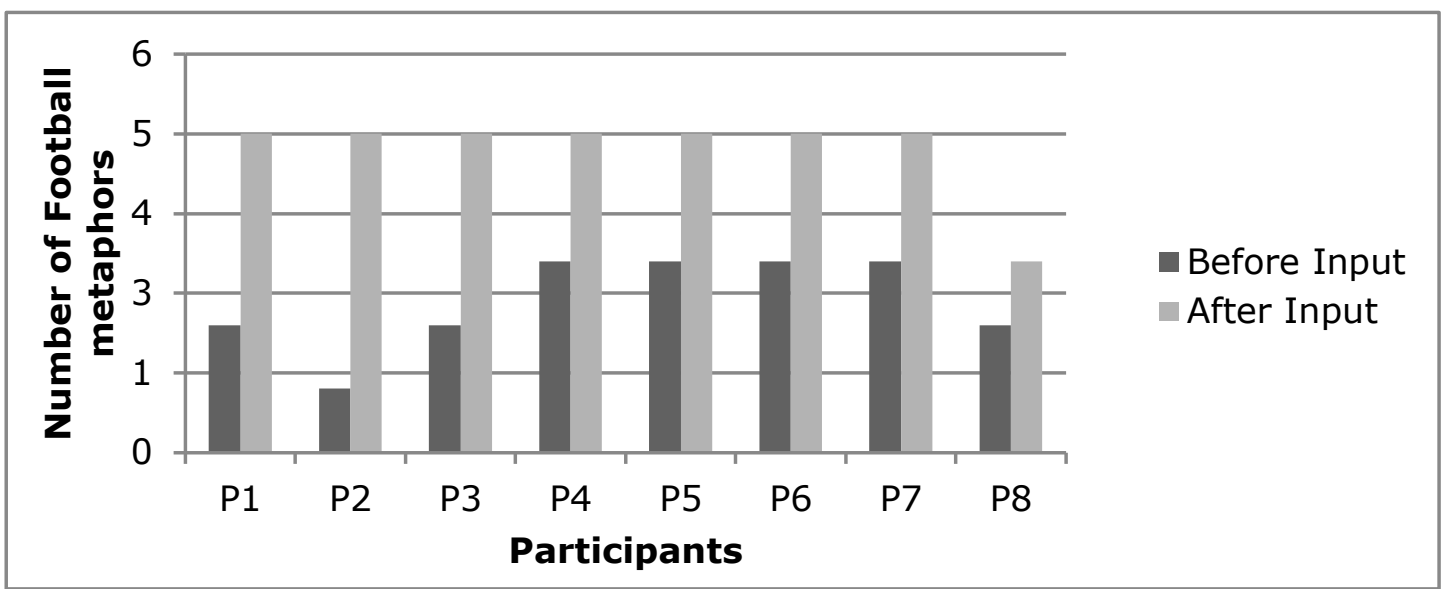

Figure 3. Individual acquisition of Football metaphors

Larsen-Freeman (2006) suggests that learners acquire expressions through negotiating with their own individual interlanguage system. The system self-organises the inputs that the learners get depending on various individual factors. In this case, 3 learners had some familiarity with the two sports. They had watched one game of American Football at the Oklahoma State University stadium and had talked about baseball with their American friends. As a result, the data showed different rates of comprehension of the metaphors by the participants. Participant 4,7 and 8 admitted that they had been to a football game at OSU Stadium, and due to that it was easier for them to understand the sports conventions and metaphorical expressions related to football. None of the participants had any previous exposure to baseball. However, all of my participants, except for participant 8 were interested in baseball. If we take the case of participant 2, his general information questionnaire revealed that he was interested in cricket and soccer (known as football in most parts of the world). He had never been exposed to any baseball or football games in the United States. Therefore, when I, first gave them the questionnaire, his baseball metaphor prediction was 0 out of 5 and football metaphor understanding was 1 out of 5. Even though his English level was lower than most other participants, he was very interested to learn these expressions as a way to develop his fluency and native-like command over metaphorical expressions. His data showed that out of the five football metaphors, he could only predict the meaning of "to score a touchdown" without any input. When asked about it, he said that it was a guess based on his knowledge of the word "score." After the end of four weeks he showed a staggering improvement and had predicted 10 out of 10 metaphors correctly.

On the other hand, participant 8 showed a clear lack of motivation when the research was being conducted. He said he was not interested in baseball or football and was not at all keen on developing metaphorical expressions. He stressed that he was in the US to do research, and not to gain fluency in English. When the rules of the game were being explained, he seemed absent-minded and preoccupied with his own research. If we look at his results, we see that before the input he had predicted 1 in the baseball category and 2 in the football category correctly. After receiving input, despite his lack of motivation, he successfully predicted 5 and 3 in the categories respectively despite his lack.

The results varied also due to the frequency of the use of the metaphors. The metaphors showed different frequency rate in the COCA database. The frequencies, if looked at, explain why some metaphors were easier to access for non-native speakers and cultural outsiders of English and why the other ones were not. We have found that the frequency of the expressions in COCA was: major league (3650), running back (3213), kick-off (598), off base (228), to hold the line (168), home stretch (111), to score a touchdown (100), go to bat for (47), hard hitter (20) and dancing at the end zone $(0)$. In this research project, the results show that even before they received controlled input, 6 out of the 8 participants could correctly predict the meaning of the highest frequency metaphor "major league", whereas, none of the participants even attempted to predict a lower frequency metaphor "home stretch" in the first set of data. However, once explained 6 out of 8 participants predicted the meaning rightly. The lowest frequency was showed for "Dancing at the end zone" $(0)$ and like the previous one, none of the participants even attempted to answer it before the input was available to them. After the participants received controlled input, all 8 participants retained the metaphors until the time they were tested again. All of them had predicted it correctly at the second round of MAT. 
This indicates that the participants not only self-arranged their interlanguage system as they are encountered new words and expressions; they are also constantly negotiated the meanings with the already existing domains and mental lexicon. The linguistics domains are interconnected and interdependent, therefore, we constantly borrow from one domain to another. In the case of sports metaphors, we see that the participants are also doing the same thing. Even though my initial hypothesis was that the participants would not be familiar with the American Football expressions, they show a deeper understanding of the conventions and expressions than they did when they were predicting about baseball expressions. Cooper (1999) had shown in a previous study that L2 speakers, largely employ three major strategies-guessing from context, discussing, analyzing the idiom, and using the literal meaning of the idiom to access metaphors. We have seen similar strategies being employed by the participants.

During the debriefing, I had asked the group why they were interested in learning about American football. They replied that living in Stillwater and going to Oklahoma State University have made them increasingly interested in football. They also encountered football news very frequently in the campus newspaper. Since football was a part of the campus life at OSU, learning about it may help them integrate into the new society. The motivation shows that "epistemic correspondence" (Kovecses \& Szabo, 1996) is a part of the overall metaphor learning process. In addition to that, transactions are taking place between several domains that are at work when a participant is acquiring new items of language and culture.

Even though each of my participants showed a different trajectory of development, they all were put through the trifold strategy of Boers (2004), i) by making the figurative language literal (or original), ii) by employing learners' problem-solving skills for retention of the metaphors, and iii) grouping idioms under a broad metaphoric theme for clearer understanding. I conclude that motivation worked as a strong factor in their acquisition of the metaphors. This paper also repositions language acquisition as an outcome of a socio-cognitive context. It reaffirms that language acquisition happens in a social context with the presence of sufficient linguistic input.

\section{Conclusion}

In this study, I took the approach of frame semantics in order to explain the acquisition of metaphors. Chales Filmore (2006) explains that lexical items are parts of a whole frame and their meaning is constructed based on our knowledge of the interconnecting networks between the frames. In this study, my attempt was to give the participants an idea about the two specific domains of Baseball and American Football. And from there develop their awareness about idiomatic usage of concepts from within those domains.

In this study, my attempt was to give the participants an idea about the two specific domains of Baseball and American Football and from there, develop their awareness about idiomatic usage of concepts from within those domains. I was also interested to see how whether the learners could negotiate the meaning of new metaphors from already existing domains.

The results of this research showed that "sufficient and controlled input" facilitate better acquisition and retention by adult speakers as mentioned by Tomasello (2003). Almost all the participants were interested in sports and some of the expressions that they had encountered during the research had corresponded to some of their background knowledge of some other domains. However, making the metaphors turn into real life sports conventions in their head, and then having them perform the task of guessing and at the end having them guess their meaning from a metaphorical context had given them a lot of controlled input and all of them show a trajectory of development. I am aware of the drawback of this study as being limited to a shorter time frame. A longitudinal study would explain more the acquisition and the retention of the metaphors that the learners received input for in this study. I intend to focus on a particular learner for longitudinal study in the future.

\section{Acknowledgements}

The author would like to thank the editor for their valuable time and advice. 


\section{References}

Boers, F. (2004). Expanding learners' vocabulary through metaphor awareness: What expansion, what learners, what vocabulary. Cognitive linguistics, second language acquisition, and foreign language teaching, 211-232.

Boers, F., \& Demecheleer, M. (2001). Measuring the impact of cross-cultural differences on learners' comprehension of imageable idioms. ELT journal, 55(3), 255-262.

Cooper, T. C. (1998). Teaching idioms. Foreign language annals, 31(2), 255-266.

Cooper, T. C. (1999). Processing of idioms by L2 learners of English. Tesol Quarterly, 33(2), 233-262.

Fernando, C., \& Flavell, R. H. (1981). On Idiom: Critical View and Perspectives (Vol. 5). University of Exeter Press.

Geba, T., Hoban, N., \& Rambut, K. (2018). Descendants of Ndori clan: Lio ethnic Ende regency. International Journal of Social Sciences and Humanities (IJSSH), 2(2), 54-64.

Grant, L., \& Bauer, L. (2004). Criteria for Re-defining Idioms: Are we Barking up the Wrong Tree?. Applied linguistics, 25(1), 38-61.

Johnson, M. (1980). Metaphors we live by. Chicago: University.

Kovecses, Z., \& Szabco, P. (1996). Idioms: A view from cognitive semantics. Applied linguistics, 17(3), 326-355.

Larsen-Freeman, D. (2006). The emergence of complexity, fluency, and accuracy in the oral and written production of five Chinese learners of English. Applied linguistics, 27(4), 590-619.

Lindayani, L. R., Masri, F. A., Idul, R., \& Sawali, L. (2018). A metaphorical analysis of Kabhanti Modero to show Munanese social relations. International Journal of Linguistics, Literature and Culture (IJLLC), 4(2), 72-80.

Suryasa, I. W. (2016). Potential and actual expression in blocking system (morphology studies). International Journal of Research in Social Sciences, 6(3), 682-691.

Tomasello, M., Abbot-smith, K., Gries, S. T., \& Stefanowitsch, A. 2003 Constructing a Language: A Usage-based Theory of Language Acquisition. 